\title{
Canadian Association of Emergency Physicians Position Statement on Acute Ischemic Stroke
}

Devin Harris, MD*; Christopher Hall, MD ${ }^{\dagger}$; Kevin Lobay, MD; Andrew McRae, MD ${ }^{\dagger}$; Tanya Monroe, MD Jeffrey J. Perry, MD, MSc"; Anthony Shearing, MD**; Gabe Wollam, MD ${ }^{\dagger+}$; Tom Goddard, MD ${ }^{\ddagger \neq}$; Eddy Lang, $\mathrm{MD}^{\dagger}$

\section{EXECUTIVE SUMMARY}

The CAEP Stroke Practice Committee was convened in the spring of 2013 to revisit the 2001 policy statement on the use of thrombolytic therapy in acute ischemic stroke. The terms of reference of the panel were developed to include national representation from urban academic centres as well as community and rural centres from all regions of the country. Membership was determined by attracting recognized stroke leaders from across the country who agreed to volunteer their time towards the development of revised guidance on the topic. The guideline panel elected to adopt the GRADE language to communicate guidance after review of existing systematic reviews and international clinical practice guidelines. Stroke neurologists from across Canada were engaged to work alongside panel members to develop guidance as a dyad-based consensus when possible.

There was no unique systematic review performed to support this guidance, rather existing efficacy data was relied upon. After a series of teleconferences and face to face meetings, a draft guideline was developed and presented to the CAEP board in June of 2014. The panel noted the development of significant new evidence to inform a number of clinical questions related to acute stroke management. In general terms the recommendations issued by the CAEP Stroke Practice Committee are supportive of the use of thrombolytic therapy when treatment can be administered within 3 hours of symptom onset. The committee is also supportive of system-level changes including pre-hospital interventions, the transport of patients to dedicated stroke centers when possible and tele-health measures to support thrombolytic therapy in a timely window.

Of note, after careful deliberation, the panel elected to issue a conditional recommendation against the use of thrombolytic therapy in the $3-4.5$ hour window. The view of the committee was that as a result of a narrow risk benefit balance, one that is considerably narrower than the same considerations under 3 hours, a significant number of informed patients and families would opt against the risk of early intracranial hemorrhage and the possibility of increased 90-day mortality that is not seen for more timely treatment. Furthermore, the frequently impaired nature of patients suffering an acute stroke and the difficulties in asking families to make life and death decisions in a highly time-sensitive context led the panel to restrict a strong endorsement of thrombolytic to the 3 hour outermost limit. The committee noted as well that Health Canada has not approved a thrombolytic agent beyond a three hour window in acute ischemic stroke.

\section{CAEP POSITION}

Stroke systems of care should be developed to optimize prehospital triage and transport to designated stroke centers. We recommend intravenous thrombolysis for eligible acute ischemic stroke patients who can be treated within three hours of stroke symptom onset. We suggest against intravenous thrombolysis for eligible patients who would receive treatment between 3 to

From the *St. Paul's Hospital, Vancouver, BC; †Cumming School of Medicine, University of Calgary, Calgary, AB; $¥$ University of Alberta, Edmonton, AB; §Dalhousie University, Halifax, NS; ๆUniversity of Ottawa, Ottawa, ON; **Northern Ontario School of Medicine, Thunder Bay, ON; ††Society for Rural Physicians of Canada, Mount Forest, ON; and ¥‡Dalhousie University, Annapolis Valley District Health Authority, Halifax, NS.

Correspondence to: Dr. Eddy Lang, Academic and Clinical Department Head for Emergency Medicine Cumming School of Medicine Alberta Health Services - Calgary Zone, Calgary, AB, Canada T2N 1N4; Email: eddy.lang@albertahealthservices.ca 
4.5 hours after symptom onset until further research is available. The majority of stroke patients should be managed in dedicated stroke units. Telestroke should be supported as a means of providing acute stroke expertise where local support is limited. Rural settings represent unique challenges to the safe and effective administration of thrombolytic therapy within the three hour time window. In some of these settings adherence to a 3 hour mark may not be possible.

\section{INTRODUCTION}

The management of acute ischemic stroke in emergency departments, by emergency physicians, is time sensitive and complex. Emergency physicians are increasingly responsible for the initial management of acute stroke patients and are often the only treating physician in some community and most rural settings. Recognizing the need to provide recommendations for emergency physicians throughout Canada, this position statement for thrombolysis in acute ischemic stroke seeks to update and expand upon the original position statement from 2001.

In 2001 the Canadian Association of Emergency Physicians issued a position statement regarding the use of thrombolytic therapy in acute ischemic stroke. ${ }^{1}$ The guidance issued by the twenty four emergency physician members of the CAEP committee for the administration of thrombolytic therapy in acute ischemic stroke noted that "Further evidence is necessary to support the widespread application of stroke thrombolysis outside research settings". In 2012, the CAEP Stroke Practice Committee re-established a new terms of reference promoting membership and representation from across Canada and with representation from rural, regional and urban settings. The committee forged partnerships and sought advice from leading stroke neurologists in Canada as well as the pre-hospital contingent of the Canadian Stroke Network.

The mandate of the CAEP Stroke Practice Committee members was to review specific topics in acute stroke care working in collaboration and develop guidance based on existing clinical practice guidelines and/or systematic reviews of the literature. The terms of reference for the committee were approved by the CAEP board and are available upon request. The main features involve a national representation of emergency physicians with interest and expertise in acute stroke and transparent procedures for voting recommendations into strong or weak categories. There was no attempt to conduct de novo systematic reviews or employ specific guideline methodologies develop this document and hence this guidance. The strength of recommendations and the quality of evidence designations reflect a consensus-based process emanating from the committee. Although not adhering strictly to the GRADE method of consensus, GRADE terminology was followed as a template for developing these recommendations. ${ }^{2}$ Conflicts of interest were addressed on early teleconferences and were intended to be supplemented by ICJME declarations as publication approached. There are no relevant pharmaceutical conflicts although many of the panellists were selected because they hold "stroke" portfolios in their region, some of which are remunerated. There were no specific voting procedures invoked and hence no need to recuse panellists. All decisions were reached by consensus and no panellist was excluded from any discussion.

The first task of the CAEP Stroke Practice Committee was to review the current literature and published guidelines for the management of acute ischemic stroke patients, including thrombolysis with the intent of formulating recommendations. This committee was composed of 10 emergency physicians, from various practice settings across Canada, who initially met through teleconference in September 2012. Key topics of acute ischemic stroke management identified by the Committee were: Stroke Systems of Care; Emergency Medical Services (EMS) Considerations; Thrombolytic Therapy within 3 Hours of Stroke Symptom Onset; Thrombolytic Therapy within 3 to 4.5 Hours of Stroke Symptom Onset; Optimal Post Thrombolysis Monitoring/Transitions in Care, and; Telestroke. Reviews were performed from September 2012 to May 2013, through online means and teleconferences. An in-person meeting was held in June 2013 to review recommendations and discuss elements of the draft document. Each topic is organized with an introduction to the problem, a brief literature review, and a summary of recommendations. The Committee relied in part upon the Canadian Best Practices for Stroke Care for the literature review and evidence. ${ }^{3}$

\section{A. STROKE SYSTEMS OF CARE}

\section{Introduction to the Problem}

The optimal management of patients with acute stroke and transient ischemic attack (TIA) is time sensitive, 
and becoming increasingly complex. However, barriers exist to providing optimal stroke care, from prehospital to inpatient settings. In the prehospital setting, access to thrombolytic therapy for acute ischemic stroke (AIS) is hindered by time delays caused in part by a lack of public awareness of stroke symptoms, delayed assessment and transport by emergency medical systems (EMS) personnel, and transport to hospitals that do not administer tPA. Within emergency departments, inefficiencies in triage, assessment, imaging and referral may impact time to definitive care. In hospital, lack of specialized stroke care impacts patient outcomes and hospital length of stay. Finally, delays in assessment and treatment of TIA patients within emergency departments may have an impact on the risk of recurrent stroke. These barriers highlight the need for organized stroke systems of care, from coordinated pre-hospital assessment and destination protocols to organized inpatient (stroke unit) care.

\section{Review of the Literature}

Evidence from controlled trials shows the effectiveness of a variety of interventions for acute stroke and TIA. Thrombolysis with recombinant tissue plasminogen activator within 3 hours of stroke symptom onset has strong evidence for its effectiveness. ${ }^{4}$ Once EMS dispatch is accessed, systems must be in place to rapidly assess and transport AIS patients to emergency departments with thrombolysis capability. Evidence now shows that coordinated prehospital stroke strategies, including prehospital notification and targeted transport to dedicated stroke centers, results in shorter times to thrombolysis and increased proportions of stroke patients receiving thrombolytic therapy. ${ }^{5}$ However, not all sites have this capability; administration of thrombolysis requires efficient ED systems, prompt 24-hour imaging capability, and access to clinicians with expertise in stroke. Integrated telestroke systems provide access to stroke expertise for those centers that do not have local resources. ${ }^{6}$ In the inpatient setting, there is strong evidence that patients managed on organized inpatient stroke units have much better clinical outcomes. ${ }^{7}$ Stroke unit care reduces the likelihood of death and disability in men and women of any age with mild, moderate, or severe stroke by as much as $30 \% .{ }^{8}$ Additionally, rapid assessment and management of patients with TIA also has strong evidence to its effectiveness. TIA patients who are rapidly assessed and treated in coordinated secondary prevention systems have a lower rate of stroke within 90 days. $^{9}$

\section{Recommendations}

1. Prehospital triage and transportation protocols should identify facilities locally or regionally designated to receive acute stroke patients and bypass more proximal facilities in favor of transporting to these sites, assuming the absence of other immediately life-threatening conditions (STRONG RECOMMENDATION, MODERATE QUALITY EVIDENCE). ${ }^{5,7,10,11}$

2. Emergency departments that treat acute stroke patients should have "Code Stroke" protocols or stroke teams to rapidly assess, image and treat patients with the highest priority (STRONG RECOMMENDATION, MODERATE QUALITY EVIDENCE). ${ }^{10}$

3. Designated stroke centers should be identified within regions to receive acute stroke patients; these centers should have organized inpatient (stroke unit) services (STRONG RECOMMENDATION, STRONG QUALITY EVIDENCE). ${ }^{7,8,12}$

4. Telestroke networks should be established to provide access to stroke expertise for centers with limited local acute stroke resources as well as expertise in CT scan interpretation to exclude contraindications to lytic therapy (STRONG RECOMMENDATION, MODERATE QUALITY EVIDENCE). ${ }^{6,12}$

5. Coordinated secondary prevention systems of care for TIA patients should be established (MODERATE RECOMMENDATION, MODERATE QUALITY EVIDENCE). ${ }^{9}$

\section{B. EMERGENCY MEDICAL SYSTEMS CONSIDERATIONS}

\section{Introduction to the Problem}

Clinical outcomes in patients with acute ischemic stroke are closely tied to the time delay between the onset of symptoms and the administration of thrombolytic therapy. For this reason, the approach to acute stroke treatment has come to be conceptualized in much the same fashion as with acute coronary syndromes, following the maxim that 'time is brain'. Most of the time delay to administering thrombolysis elapses prior to the patient arriving in the Emergency Department. ${ }^{13}$ Accordingly, a strong emphasis needs to be placed on identifying the factors contributing to prehospital 
delays in accessing definitive therapy. Finding ways to diminish or eliminate these factors represents a significant opportunity to improve the outcomes experienced by patients with acute ischemic stroke.

\section{Review of the Literature}

The single greatest contributor to prehospital delays in obtaining acute stroke treatment can be attributed to the time taken for the patient or bystanders to initiate access to emergency medical care. This hesitation may be due not only to a lack of knowledge regarding the symptoms of stroke, but also to a failure to recognize these symptoms as constituting a medical emergency. Various public education campaigns have focused not only on increasing public knowledge of the symptoms of stroke but also on the need to activate EMS at the earliest possible moment. ${ }^{14}$ These efforts have demonstrated limited degrees of success in reducing thrombolytic times, making the search for effective methods of public education a high priority in the ongoing effort to reduce prehospital delays in stroke care. Fortunately, greater clinical successes have been derived from efforts to streamline prehospital diagnosis, triage and transportation of suspected acute stroke patients. The use of standardized stroke assessment instruments has been demonstrated to improve stroke identification in the field by prehospital personnel. ${ }^{14}$ Implementing these tools as part of a coordinated prehospital stroke strategy, including prehospital notification and targeted transport to dedicated stroke centers, results in shorter times to thrombolysis and increased proportions of stroke patients receiving thrombolytic therapy. ${ }^{5,15}$ Prehospital protocols assigning stroke patients to the highest transport priority level do not appear to negatively influence transport times for other medical emergencies. ${ }^{15}$ The use of such strategies is likely one of the core reasons for the observed decline in prehospital delays to accessing thrombolytic therapy over the past decade. ${ }^{13}$ Future directions may include mobile 'stroke units', which have been shown to sharply reduce thrombolysis times in emergency medical systems outside of North America. ${ }^{16}$

\section{Recommendations}

1. Emergency medical dispatchers should triage calls identified as suspected acute stroke with the highest transport priority level possible (STRONG RECOMMENDATION, HIGH QUALITY EVIDENCE). ${ }^{15}$
2. Initial priorities for prehospital providers assessing suspected acute stroke patients include standard treatment of emergent airway, breathing, and circulatory compromise, followed by establishing a firm time of onset of symptoms (to be set at the time last seen to be normal by bystanders if symptoms occurred on waking from sleep or precise time cannot otherwise be accurately ascertained) and obtaining a point-of-care blood glucose level to rule out hypoglycemia as a stroke mimic (STRONG RECOMMENDATION, MODERATE QUALITY EVIDENCE) ${ }^{10}$

3. Prehospital assessment of suspected acute stroke patients should employ a validated and standardized clinical tool such as the Los Angeles Prehospital Stroke Scale which can be rapidly completed in the field (STRONG RECOMMENDATION, MODERATE QUALITY EVIDENCE). ${ }^{5,10,11}$

4. Prehospital triage and transportation protocols should identify facilities locally or regionally designated to receive acute stroke patients and bypass more proximal facilities in favour of transporting to these sites, assuming the absence of other immediately lifethreatening conditions (STRONG RECOMMENDATION, MODERATE QUALITY EVIDENCE). ${ }^{5,10}$

5. Identification of suspected acute stroke by prehospital providers should prompt immediate prenotification of the destination facility (STRONG RECOMMENDATION, MODERATE QUALITY EVIDENCE). $5,10,14$

\section{TELESTROKE}

\section{Introduction to the Problem}

Optimal acute ischemic stroke care is aided by timely consultation with stroke neurology specialists. Emergency physicians may draw upon their expertise for assistance with image interpretation, diagnosis and therapy. Telestroke is defined as "the use of audio (including telephone), video, and other telecommunications and electronic information processing technologies for the transmission of information and data relevant to the diagnosis and treatment of acute stroke." ${ }^{28}$ Since their inception in the early 1990's, telestroke programs have linked Canadian emergency physicians with neurologists who have focused expertise in stroke care. 


\section{Review of the Literature}

A systematic review of literature pertaining to telestroke utilization was published by the Canadian Agency for Drugs and Technologies in Health in January 2008. ${ }^{28}$ This extensive English-language database review included 22 relevant recent studies. No randomized controlled trials were published. Original research articles consisted of descriptions of existing telestroke programs and assessments of patient outcomes.

Functional disability and mortality rates in telestroke treated patients are similar to those in patients treated at tertiary care centres. ${ }^{29}$ Similarly, symptomatic hemorrhagic transformation rates are similar when comparing telestroke services with face-to-face stroke care by stroke neurologist. ${ }^{30}$ It is important to note that no randomized studies have been completed. Comparisons are generally made to historical controls, without case matching. When compared to telephone consultation alone, videoconferencing improves diagnostic accuracy and also appears to be associated with a reduction in 6-month mortality. ${ }^{30}$

Pre and post-intervention studies report significantly improved access to thrombolysis following telestroke implementation. Treatment within networked hospitals (those linked by a formal stroke program) is associated with improved outcomes. The incorporation of rural hospitals into a stroke network illustrates the potential for provision of specialized acute stroke care to sites with significant geographic separation from an urban centre. Door to needle times with videoconference utilization were good, with an average of 76 minutes reported in one study and comparable times described in others.

In neurosurgical emergencies, videoconferencing has been shown to increase initial costs, relative to telephone conferencing alone. It is not clear that is the case in acute stroke assessment, however, where telestroke consultation may actually directly reduce costs via a reduction in unnecessary patient transport costs. In addition, Quality Adjusted Life Year analysis indicates that telestroke consultation is highly cost-effective. ${ }^{31,32}$ There is a decrease in mean hospital length of stay at centers supported by telestroke, reflecting improved patient outcomes associated with earlier diagnosis and treatment. Although relatively few studies address this issue, patients and healthcare providers report high levels of satisfaction. ${ }^{33,34}$
Over 40 telestroke programs worldwide are currently in existence. ${ }^{32}$ In Canada, formal Telestroke programs exist in both Alberta and Ontario. Pilot programs in British Columbia have been trialed, but are currently unfunded. Programs described in the literature differ in terms of staffing, catchment, and intervention measurement. Most consist of a multidisciplinary team of physicians (neurologists/stroke specialists,), nurse practitioners and nurses, information technology experts, program coordinators and managers. The Canadian Stroke Strategy has advocated for expansion of telestroke services on a regional basis across Canada. ${ }^{35}$

\section{Recommendations}

1. Telestroke should be incorporated into care for patients with acute stroke at centers wherein stroke neurologists are not available on-site and this should be a prerequisite for thrombolytic therapy administration in rural settings (STRONG RECOMMENDATION, MODERATE QUALITY EVIDENCE).

2. The incorporation of video conferencing in the telehealth consultation is recommended (STRONG RECOMMENDATION, HIGH QUALITY EVIDENCE).

\section{THROMBOLYTIC THERAPY WITHIN 3 HOURS OF STROKE} SYMPTOM ONSET

\section{Introduction to the Problem}

Beginning in the mid-2000s, recombinant tissue plasminogen activator (rt-PA) was approved by various regulatory agencies for the treatment of acute ischemic stroke within three hours of symptom onset. These approvals followed the publication of the National Institutes of Neurologic Disorders and Stroke (NINDS) trial, ${ }^{17}$ which was the first large trial to demonstrate improvements in patient outcomes with fibrinolytic therapy for acute stroke. Since the NINDS trial, other large RCTs have attempted to replicate this finding. ${ }^{4}$ Summarizing the literature describing the safety and efficacy of fibrinolytic therapy for acute stroke is challenging because of the heterogeneity in study design and outcomes. Nonetheless, administration of rt-PA within three hours of symptom onset improves functional outcomes of patients with acute ischemic stroke without an increase in overall mortality. ${ }^{4,19,20}$ 


\section{Summary of Evidence}

Seven trials either specifically addressed the efficacy of rt-PA in stroke within three hours of symptom onset or provided data on a subgroup of patients receiving therapy within three hours of symptom onset.

For patients treated within three hours of onset of symptoms, there is no evidence for a 90-day mortality benefit to rt-PA therapy compared to placebo (pooled OR $1.00,95 \%$ CI $0.81-1.24)^{4}$

The risk of symptomatic intracranial hemorrhage within 7 days is substantially higher in patients treated with rt-PA within three hours of onset $(2.8 \%)$ compared to patients randomized to placebo $(0.3 \%)$ (pooled OR 10.9, 95\% CI 2.92-46.4, NNH 40). ${ }^{4}$ The rate of symptomatic intracranial hemorrhage has been lower in other observational studies. ${ }^{21}$

Fibrinolytic therapy administered within three hours of symptom onset increases the probability of survival with a favourable neurologic outcome (pooled OR 1.75, 95\% CI 1.35-2.27.86, NNT 10). ${ }^{4}$ This favourable effect appears to be independent of age, including patients aged 80 and older. ${ }^{4}$

Pooled data from several clinical trials suggest an important relationship between time from symptom onset to treatment and outcome. Patients treated with rt-PA within 90 minutes to symptom onset were more likely to have a favourable neurologic outcome compared to those treated from $90-180$ minutes to treatment onset. ${ }^{22,23}$

Although the risks of fibrinolytic therapy are substantial, and there does not appear to be evidence of a mortality increase, the moderate probability of better neurologic outcomes for survivors suggests an overall benefit to rt-PA therapy for acute ischemic stroke, if given within three hours of symptom onset.

Guidelines from the American Heart Association and a Clinical Policy Statement from the American College of Emergency Physicians recommend rt-PA treatment for eligible stroke patients who can be treated within three hours of symptom onset. ${ }^{19,20}$

\section{Recommendations}

1. Patients with acute ischemic stroke whose neuroimaging excludes contraindications, and who can be treated within three hours of symptom onset, should be offered rt-PA with the goal of improving functional outcome (STRONG RECOMMENDATION, HIGH QUALITY EVIDENCE). ${ }^{4,18,22}$
2. Stroke patients meeting eligibility criteria for thrombolytic therapy should be treated as rapidly as possible, with a target door-to-needle time of less than 60 minutes (STRONG RECOMMENDATION, MODERATE QUALITY EVIDENCE). ${ }^{19,20,22}$

3. Due to limited resources and practical constraints, the administration of thrombolytic therapy within 3 hours in rural hospital and may not be feasible and hence not recommended in all of these settings but should fall to the discretion of the local decisionmaking team (WEAK RECOMMENDATION, LOW QUALITY EVIDENCE).

\section{E. THROMBOLYTIC THERAPY WITHIN 3-4.5 HOURS OF STROKE SYMPTOM ONSET}

\section{Introduction to the Problem}

The administration of thrombolytic therapy in the management of acute ischemic stroke is exquisitely time-sensitive. Consistently, across trials it is evident that there is a direct correlation between early administration and improved outcomes with reduced risk of hemorrhage. The efficacy of fibrinolytic therapy when administered within three hours carries favorable risk benefit considerations. Whether these considerations extend to the 4.5 hour window, or beyond has been the subject of considerable study and debate.

\section{Summary of Evidence}

A pooled analysis of all trials involving acute ischemic stroke patients treated in the 3.0 to 4.5 hour time window was published by Emberson et al. in 2014. ${ }^{4}$ Combing data from 7 studies that included 2768 patients, the likelihood of a favorable outcome was higher if patients were randomized to rt-PA. Specifically the review reported an OR of 1.26 (95\% CI 1.05-1.51) for a favorable outcome as defined by a modified Rankin Score of 0 or 1, equivalent to an NNT of 20. There was no difference in 90-day mortality noted but the $95 \%$ confidence intervals around the point estimate OR of 1.14 ranged from 0.95 to 1.36 , the latter figure showing significant imprecision and no difference in mortality, but a direction of effect towards a mortality increase with rt-PA in a dose-response relationship between time to treatment and 90-day mortality, suggesting a potential for increased mortality in those treated beyond 3 hours. 
Included in this most recent systematic review is patient-level data from the IST-3 trial. ${ }^{4,24,25}$ IST-3 randomized over 3000 patients to rt-PA or placebo among patients presenting receiving treatment in a variety of time windows up to 6 hours after onset of stroke. With regards to the primary outcome of functional recovery with an Oxford Handicap Score or 0-2, the 1177 patients treated in the $3-4.5$ hour time window did not do better with thrombolytic therapy compared to placebo; OR 0.73 (95\% CI 0.5-1.07). IST-3 suggested a 7-fold increase in symptomatic ICH associated with thrombolytic use (7\% vs. $1 \%)$ as well as an expected increase in early mortality but no difference in disability or mortality at 6 months for those treated at 3-4.5 hours.

\section{Recommendations}

1. Thrombolytic therapy for acute ischemic stroke patients should not be routinely offered for the treatment of acute ischemic stroke for patients if administered beyond three hours of stroke symptom onset (WEAK RECOMMENDATION, MODERATE QUALITY EVIDENCE).

2. The administration of thrombolytic therapy for acute ischemic stroke beyond 3 hours from stroke symptom onset should be restricted to specialized stroke centers with advanced imaging capabilities or as part of a research protocol (WEAK RECOMMENDATION, LOW QUALITY EVIDENCE).

In making these recommendations we place a higher value on avoidance of short term harm as a result of a medical intervention in the face of uncertain benefit in functional outcome in the longer term. We are also of the view that the risks and benefits of rt-PA administration beyond the three hour window cannot be properly explained and considered by patients and their loved ones in the rushed environment associated with the acute stroke context.

Of note and in keeping with a weak or conditional recommendation, thrombolytic therapy may be offered to patients beyond the 3-hour window if the patient is thought to be of low hemorrhage risk and advanced neuro-imaging suggests that there is salvageable brain. As a paucity of evidence for benefit exists in this patient population, consultation with subspecialty neurologists, and enrollment in a clinical trial are strongly advised. As such, this guideline opens the door for physicians to administer thrombolysis in a subgroup that may benefit as deemed by the treating team.

\section{F. OPTIMAL POST THROMBOLYSIS MONITORING/} TRANSITIONS IN CARE

\section{Introduction to the Problem}

The largest population-based impact for improving mortality and morbidity post-stroke is to provide care for stroke patients on dedicated stroke units. Stroke units are defined as, "a specialized, geographically defined hospital unit dedicated to the management of stroke patients." ${ }^{3}$ Overwhelming high-quality RCT evidence has demonstrated that caring for patients with stroke on a stroke unit decreases the likelihood of death or disability by as much as $30 \%$ in patients across the full spectrum of stroke severity. ${ }^{3,26}$

Canadian benchmarks, based on consensus opinion, have indicated that stroke patients should be transferred from the Emergency Department to a stroke unit within 3 hours of presentation. ${ }^{3}$ This benchmark may not yet be consistently achievable in many Canadian hospitals. Stroke patients who have received tPA form a special group in this regard, as careful monitoring of this patients is needed. Consequently, post-thrombolysis monitoring may need to occur in the ED setting for a period of several hours or more.

Approximately $25 \%$ of all stroke patients may deteriorate in the 24-48 hours post stroke. ${ }^{10}$ One goal of hospital admission post-stroke is to observe for changes in patient condition that may herald the need for further intervention. In the subset of patients who have received tPA, minimizing the risk of and monitoring for complications is an important aspect of this care.

\section{Review of the Literature}

There is strong consensus in the literature about post TPA monitoring and care. ${ }^{3,10,27}$ Adhering to best practices has a significant benefit to patient outcome. Specifically, attention to details such as temperature, blood glucose and swallowing dysfunction are "low tech / high yield" interventions in the stroke population and have significant mortality benefit. ${ }^{27}$ Monitoring for and minimizing the risk of complications starts in the $\mathrm{ED}$ and continues as the patient transitions to inpatient settings.

Intracranial hemorrhage is an uncommon but important complication of thrombolytic therapy. Outcomes are catastrophic and generally fatal and not amenable to neurosurgical intervention. 


\section{RECOMMENDATIONS}

\section{A) Post thrombolysis recommendations}

1. Following intravenous thrombolysis, vital signs (including neurovitals) should be monitored q15mins $\times 2$ hours, then $\mathrm{q} 30$ mins $\times 6$ hours, then $\mathrm{q} 1 \mathrm{~h}$ $\times 24$ hours (STRONG RECOMMENDATION, LOW QUALITY EVIDENCE).

2. Avoidance of arterial or central venous puncture in the first 24 hours following intravenous thrombolysis is recommended (BEST PRACTICE STATEMENT).

3. Antithrombotic drugs (antiplatelet and anticoagulant agents) should be avoided for 24 hours after intravenous thrombolysis administration (WEAK RECOMMENDATION, LOW QUALITY EVIDENCE).

4. Patients who have received intravenous thrombolysis should be monitored closely for signs of airway compromise, which may be an indication of hemilingual angioedema. This is especially true in the case of patients who have been taking angiotensin converting enzyme inhibitors (ACEI). Emergency Department staff should be trained in the management of hemilingual angioedema (BEST PRACTICE STATEMENT).

5. Onsite neurosurgical support is not required for managing post-thrombolytic care (WEAK RECOMMENDATION, LOW QUALITY EVIDENCE).

\section{B) General recommendations}

1. While in the Emergency Department, patients should have continuous cardiac monitoring to assess for atrial fibrillation. Ideally, continuous cardiac monitoring should continue for at least 24 hours post intravenous thrombolysis (STRONG RECOMMENDATION, MODERATE QUALITY EVIDENCE).

2. Evidence about the optimal management of blood pressure in the hyperacute phase of stroke, or in the immediate post-thrombolysis period is limited. A consensus view, based on blood pressure treatment parameters prior to and during thrombolysis administration, would suggest that blood pressure be lowered if $>185$ systolic or $>105$ diastolic. Blood pressure should be lowered only by $15 \%$ acutely and no more than $25 \%$ over the first 24 hours. Using easily titrated agents (labetolol, nitroprusside, etc) to control blood pressure in the first 24 hours is reasonable ${ }^{3,10,27}$ (WEAK RECOMMENDATION, LOW QUALITY EVIDENCE).

3. All patients with acute stroke should have a swallowing assessment undertaken prior to administration of any medication or nutrition by mouth. The use of nasogastric (NG) tubes should be avoided for up to 24 hours after intravenous thrombolysis administration, but could be inserted after 12 hours if necessary for medications that cannot be provided using another route. Hydration with IV fluids (normal saline) should be instituted (BEST PRACTICE STATEMENT).

4. Maintenance of euglycemia has been associated with better neurological outcomes in stroke. Routine, repeated measurement of blood glucose while in the Emergency Department is recommended, as is avoidance of glucose containing IV fluids (STRONG RECOMMENDATION, MODERATE QUALITY EVIDENCE).

5. Urinary catheters should not be placed following tPA administration, and should be generally avoided in all patients with stroke, as they are a potential source of infection (STRONG RECOMMENDATION, MODERATE QUALITY EVIDENCE).

6. Temperature should be monitored as part of routine vital sign assessments, every four hours for first 48 hours. For temperature greater than $37.5^{\circ}$ Celsius, increase frequency of monitoring, initiate temperaturereducing measures, investigate possible infection such as pneumonia or urinary tract infection (BEST PRACTICE STATEMENT).

Acknowledgments:: The authors would like to thank Charles Duffy, Michael Ertel, Tony Herd, Ghosh Indy, and Santiago Santa-Cruz for their contributions.

\section{SUPPLEMENTARY MATERIALS}

To view Supplementary Materials for this article, please visit http://dx.doi.org/10.1017/cem.2015.26

\section{REFERENCES}

1. Canadian Association of Emergency Physicians Committee on Thrombolytic Therapy for Acute Ischemic Stroke. Thrombolytic therapy for acute ischemic stroke. CFEM 2001;3(1):8-12.

2. Guyatt GH1, Oxman AD, Vist GE, Kunz R, Falck-Ytter Y, Alonso-Coello P, Schünemann HJ; GRADE Working Group. GRADE: an emerging consensus on rating quality 
of evidence and strength of recommendations. BM7 2008;336(7650):924-6, doi: 10.1136/bmj.39489.470347.AD.

3. Stroke Rehabilitation Unit Care. Canadian Stroke Best Practice Recommendations, 4th Edition, Section 5.2.1; 2012-2013. Available at: http://www.strokebestpractices.ca/ index.php/stroke-rehabilitation/stroke-rehabilitation-unitcare-2/ (accessed February 23, 2015).

4. Emberson J, Lees KR, Lyden P, et al; for the Stroke Thrombolysis Trialists' Collaborative Group. Effect of treatment delay, age, and stroke severity on the effects of intravenous thrombolysis with alteplase for acute ischaemic stroke: a meta-analysis of individual patient data from randomised trials. Lancet 2014;384(9958):1929-35.

5. Gladstone DJ, Rodan LH, Sahlas DJ, et al. A citywide prehospital protocol increases access to stroke thombolysis in Toronto. Stroke 2009;40(12):3841-4.

6. Zaidi SF, Jumma MA, Urra XN, et al. Telestroke-guided intravenous tissue-type plasminogen activator treatment achieves a similar clinical outcome as thrombolysis at a comprehensive stroke center. Stroke 2011;42(11):3291-3.

7. Bray BD, Ayis S, Campbell J, et al. Associations between the organization of stroke services, process of care, and mortality in England: prospective cohort study. BMF 2013; 346:f2827.

8. Stroke Unit Trialists' Collaboration. Organised inpatient (stroke unit) care for stroke. Cochrane Database of Systematic Reviews 2013;9:CD000197, doi: 10.1002/14651858.

9. Rothwell PM, Giles MF, Chandrateva A, et al. Effect of urgent treatment of transient ischaemic attack and minor stroke on early recurrent stroke (EXPRESS study): a prospective population-based sequential comparison. Lancet 2007;370(9596):1432-42.

10. Adams HP Jr, del Zoppo G, Alberts MJ, et al. Guidelines for the early management of adults with ischemic stroke: a guideline from the American Heart Association/American Stroke Association Stroke Council, Clinical Cardiology Council, Cardiovascular Radiology and Intervention Council, and the Atherosclerotic Peripheral Vascular Disease and Quality of Care Outcomes in Research Interdisciplinary Working Groups: the American Academy of Neurology affirms the value of this guideline as an educational tool for neurologists. Stroke 2007;38(5):1655-711.

11. Brandler ES, Sharma M, Sinert RH, Levine SR. Prehospital stroke scales in urban environments: a systematic review. Neurology 2014;82(24):2241-9.

12. Schwamm LH, Pancioli A, Acker JE III, et al. Recommendations for the Establishment of Stroke Systems of Care: Recommendations from the American Stroke Association's Task Force on the Development of Stroke Systems. Circulation 2005;36(3):690-703.

13. Evenson KR, Foraker RE, Morris DL, Rosamond WD. A comprehensive review of prehospital and in-hospital delay times in acute stroke care. 7 Stroke 2009;4(3):187-99.

14. Kue R, Steck A. Prehospital diagnosis and management of patients with acute stroke. Emerge Med Clin N Am 2012;30: (3):617-35.

15. Baldereschi M, Piccardi B, Di Carlo A, et al. Relevance of prehospital stroke code activation for acute treatment measures in stroke care: A review. Cerebrovasc Dis 2012; 34(3):182-90.
16. Berglund A, Svensson L, Sjostrand C, et al. Higher prehospital priority level of stroke improves thrombolysis frequency and time to stroke unit. Stroke 2012;43(10):2666-70.

17. Walter S, Kostopoulos P, Haass A, et al. Diagnosis and treatment of patients with stroke in a mobile stroke unit versus in hospital: a randomized controlled trial. Lancet Neurol 2012;11(5):397-404.

18. The National Institute of Neurologic Disorders and Stroke (NINDS) rt-PA Stroke Study Group. Tissue plasminogen activator for acute ischemic stroke. $N$ Engl 7 Med 1995;333 (24):1581-7.

19. Jauch EC, Saver JL, Adams HP Jr, et al. Guidelines for the early management of patients with acute ischemic stroke: a guideline for healthcare professionals from the American Heart Association/American Stroke Association. Stroke 2013;44(3):870-947.

20. American College of Emergency Physicians. Use of Intravenous tPA for the management of acute ischemic stroke in the emergency department. Ann Emerg Med 2013;61(2):225-43.

21. Hill MD, Buchan AM. Thrombolyisis for acute ischemic stroke: The Canadian alteplase for stroke effectiveness study. CMA7 2005;172(10):1307-12.

22. The ATLANTIS, ECASS, and NINDS rt-PA Study Group Investigators. Association of outcome with early stroke treatment: pooled. Lancet 2004;363(9411):768-74.

23. Lees KR, Bluhmki E, von Kummer R, et al. Time to treatment with intravenous alteplase and outcome in stroke: an updated pooled analysis of ECASS, ATLANTIS, NINDS, and EPITHET trials. Lancet 2010;375 (9727):1695-703, doi: 10.1016/S0140-6736(10)60491-6.

24. Lansberg MG, O'Donnell MJ, Khatri P, et al; for the American College of Chest Physicians. Antithrombotic and thrombolytic therapy for ischemic stroke: Antithrombotic Therapy and Prevention of Thrombosis, 9th ed: American College of Chest Physicians Evidence-Based Clinical Practice Guidelines. Chest 2012 Feb;141(S2):e601S-36, doi: 10.1378/chest.11-2302.

25. IST-3 collaborative group, Sandercock P, Wardlaw JM, et al. The benefits and harms of intravenous thrombolysis with recombinant tissue plasminogen activator within $6 \mathrm{~h}$ of acute ischaemic stroke (the third international stroke trial [IST-3]): a randomised controlled trial. Lancet 2012;379 (9834):2352-63, doi: 10.1016/S0140-6736(12)60768-5.

26. Anderson D, Larson D, Lindholm P, et al. Diagnosis and treatment of Ischemic Stroke. Institute for Clinical Systems Improvement (ICSI). Diagnosis and Initial Treatment of Ischemic Stroke; 2012, p. 49. Available at: https://www.icsi. org/_asset/xq13xv/Stroke.pdf (accessed February 23, 2015).

27. Middleton S, McElduff P, Ward J, Grimshaw J, Dale S, D'Este C, et al. Implementation of evidence-based treatment protocols to manage fever, hyperglycaemia, and swallowing dysfunction in acute stroke (QASC): a cluster randomised controlled trial. Lancet 2011;378(9804):1699-1706.

28. Deshpande A, Khoja S, McKibbon A, Rizo C, Jadad AR. Telehealth for acute stroke management (telestroke): Systematic review and environmental scan. Technology Overview 2008;37:1-3.

29. Demaerschalk BM, Raman R, Ernstrom K, Meyer BC. Efficacy of telemedicine for stroke: Pooled analysis of the stroke team remote evaluation using a digital observation 
camera (stroke doc) and stroke doc arizona telestroke trials. Telemed 7 E Health 2012;18(3):230-7.

30. Zaidi SF, Jumma MA, Urra XN, Hammer M, Massaro L, Reddy V, Jovin T, Lin R, Wechsler LR. Telestroke-guided intravenous tissue-type plasminogen activator treatment achieves a similar clinical outcome as thrombolysis at a comprehensive stroke center. Stroke 2011;42(11):3291-3.

31. Nelson RE, Saltzman GM, Skalabrin EJ, Demaerschalk BM, Majersik JJ. The cost-effectiveness of telestroke in the treatment of acute ischemic stroke. Neurology 2011;77(17):1590-8.

32. Muller-Barna P, Schwamm LH, Haberl RL. Telestroke increases use of acute stroke therapy. Curr Opin Neurol 2012;25(1):5-10.
33. Frey JL, Jahnke HK, Goslar PW, Partovi S, Flaster MS. tPA by telephone: Extending the benefits of a comprehensive stroke center. Neurology 2005;64(1):154-6.

34. Schwamm LH, Rosenthal ES, Hirshberg A, et al. Virtual telestroke support for the emergency department evaluation of acute stroke. Acad Emerg Med 2004;11(11): 1193-7.

35. Kalbfleisch R. Increasing access to quality stroke care by implementing TeleStroke in Canada. Canadian Stroke Network; 2014. Available at: http://canadianstrokenetwork. ca/en/wp-content/uploads/2014/08/TeleStroke_ENG-hi.pdf (accessed February 23, 2015) 